\title{
INSTRUMENTS FOR MEASURING THE PROFESSIONAL IDENTITY OF PSYCHOLOGICAL HELP PROVIDERS: RAPID LITERATURE REVIEW
}

\author{
Elīna Akmane ${ }^{1}$, Kristīne Mārtinsone ${ }^{2}$, Zane Krieḳe ${ }^{3}$ \\ 1,2,3 Rīga Stradiṇš University, Latvia
}

\begin{abstract}
The concept of professional identity is widely described, indicating that it is dynamic and variable, as it simultaneously includes the development and formation of professional identity in the dimensions of time and content. Professional identity manifests in various forms and it is measured by a variety of measurement tools.

Objective: to define what measurement tools are used to determine the professional identity of psychological help providers and to investigate the content of professional identity.

Method: a rapid literature review. A comprehensive search of scientific databases Access Medicine, Clinical Key, EBSCO e-books, Proquest Ebook Central, BMJ Journals, EBSCO host, ProQuest, SAGE journals, Wiley Online Library, Science Direct, DynaMed Plus and Cochrane Library was conducted in March, 2020 by two independent researchers therefore assuring data triangulation. Database research included the keywords "professional identity", "scale", "measure", with the Boolean operator "AND" adding professions: "art therapist", "dance and movement therapist", "drama therapist", "music therapist", "psychologist", "psychiatrist", "psychotherapist", "nurse", "social worker", and additional selection criteria - cross-sectional design studies about the measure of professional identity of different groups of psychological help providers and students, between 2009 and 2019, with full English text available. Data analysis method was narrative synthesis, which consists of three sequential steps: description of studies in logical categories, analysis of data from each of the derived categories, and synthesized conclusions for all included studies. 366 studies were identified of which 21 were included in the study.

Results: as a result, 15 categories including 50 content items were identified as the measurement aspects of professional identity.
\end{abstract}

Keywords: measurement, professional identity, psychological help providers.

To cite this article:

Akmane, E., Mārtinsone, K., \& Krieķe, Z. (2020). Instruments for Measuring the Professional Identity of Psychological Help Providers: Rapid Literature Review. Education. Innovation. Diversity, 1(1), 6-15. DOI: http://dx.doi.org/10.17770/eid2020.1.5335

\section{Introduction}

During the recent decades the focus on the mental health of the society has increased in the world. The World Health Organization defines mental health as a state of well-being in which an individual realizes his or her abilities, can cope with normal life stress, work productively and is able to contribute to the benefit of his or her community (World Health Organization: Fact Sheets "Mental health: strengthening our response", 2018). To maintain or improve the mental well-being a variety of specialists are involved which implement cross-sectional strategies and interventions in mental health. Psychological help is one of the options to support the improvement of mental health.

When describing and discussing the psychological help several different terms are used in the literature such as "psychological care", "psychological assistance", "psychological treatment". Furthermore, with the development of psychological help, a range of specialists provide psychological help within their profession. Psychological help is defined as a wide range of scientifically based methods of professional exposure, which are purposefully applied by specially trained professionals strengthen an individual's mental (psychological) well-being or reduce suffering (Mārtinsone \& Sudraba, 2019). In line with this definition authors have applied psychological help as the key term for this article. 
Latvia's binding documents in the field of psychological help identify seven groups of professionals who are involved in the provision of psychological help (Bortaščenoks et al, 2019). Psychiatrists, psychiatric nurses, psychotherapists (doctors) and art therapists (in Latvia - one profession that includes 4 arts therapies specializations - art therapists, dance and movement therapists, music therapists and drama therapists) are medical practitioners whose professional activity is directly related to the process of treatment and medical rehabilitation, within which additionally psychological help is provided. In contrast psychologists, psychotherapy specialists and social workers are not medical practitioners, but also provides psychological help within their profession.

As representatives of different professions work in the field of psychological help the topic of professional boundaries becomes more and more relevant, because often, as professional lines become more flexible, professional limits converge (Bortaščenoks et al, 2018; Ricou et al, 2018), which can lead to confusion of professional roles and responsibility. As a result, the efficiency of interprofessional cooperation may be endangered (Purvlīee et al, 2018). Researchers in the professional field point out that the understanding of professional boundaries is based on the professional identity of each professional. In turn, the process of professional identity formation is one of the most important aspects of the existence and sustainability of each profession (Spurgeon, 2012). Research into how psychological help providers form their sense of professional identity, and how this can be tested and strengthened, is essential to the development of each profession (Cowin et al, 2013).

The concept of professional identity is widely described, indicating that it is dynamic and variable, as it simultaneously includes the development and formation of professional identity in the dimensions of time and content. Already in $20^{\text {th }}$ century constructive development theorist Robert Kegan, like other researchers of individual and professional identity development, indicated that professional identity formation is a lifelong process that integrates cognitive, social and emotional abilities and begins before starting professional studies and continues throughout his professional life (Kegan, 1984).

Nowadays theoretical approaches to the formation of professional identity distinguish three domains through which professional identity is influenced and developed: individual, relational and collective identity (Vignoles et al, 2011; Skorikov et al, 2011), indicating that the individual domain includes personal characteristics, self-chosen or mandated commitments, beliefs about one's self, and the impact of multiple life experiences, the relational domain expresses the influence on identity of significant individuals, such as family members, friends, mentors, and coworkers. The collective domain reflects the impact of the social groups to which an individual belongs or wishes to join. An individual's status within the group and the group's status within society are important contributors to this identity component.

Thus, the content of a professional identity is made up of various criteria deriving from theoretical background chosen by the researcher. In an attempt to measure the professional identity of different psychological help providers, different researchers have studied different aspects of professional identity: professional values (Cowin et al, 2013; Healey \& Hays, 2012; Woo \& Henfield, 2015), belonging to, identifying with profession (Adams et al, 2006), selfreport about comfort, confidence in key practice skills (Casey et al, 2011), perceptions of professional development, personal and practice characteristics (Kazantzis et al, 2010), professional engagement (Healey \& Hays, 2012; Woo \& Henfield, 2015), intra-individual and intergroup processes connected with the development of a professional identity (Mancini et al, 2015), knowledge of the profession, attitude, professional roles and expertise, philosophy of the profession (Woo \& Henfield, 2015).

The purpose of this article is to define what measurement tools are used to study the professional identity of psychological help providers and to investigate the content of professional identity. We understand that professional identity in the process of its formation is 
formed by certain aspects. This literature review will provide clarity on what tools have been developed to measure professional identity and what aspects of professional identity are measured. By exploring these aspects, the criteria that make up the content of professional identity were identified.

\section{Method}

Data sources for this study were cross-sectional design research studies about the measurement of professional identity for different groups of the psychological help providers, completed between year 2009 and 2019 with full English text available. The research studies were quantitative aiming to measure professional identity or related phenomena, including professional self-concept, professional values, professional engagement, and professional selfidentity.

A comprehensive search of the scientific databases Access Medicine, Clinical Key, EBSCO e-books, Proquest Ebook Central, BMJ Journals, EBSCO host, ProQuest, SAGE journals, Wiley Online Library, Science Direct, DynaMed Plus and Cochrane Library was conducted in March, 2020 by two independent researchers therefore assuring data triangulation. A standardized search strategy was applied for each of the database, using the Boolean operators $A N D$ and punctuation mark". Main terms of this study were used as the key words for the search strategy: "professional identity", "scale", "measure". In the research selection process, the name of the group of psychological help providers has been added ("art therapist", "dance and movement therapist", "drama therapist", "music therapist", "psychologist", "psychotherapist", "psychiatrist", "nurse", "social worker").

After the completion of the database searches, duplicates were manually removed by the two researchers. The titles and abstracts of the remaining articles were screened according to the selection criteria for full article review to identify the applicable articles for this study. The selection criteria were either applied or described measurement instrument for professional identity or related phenomena. Each suitable article was reviewed to identify the specific measures that had been applied or described to evaluate professional identity with different groups of psychological help providers or students.

Data were analyzed in three sequential steps: 1) description of studies in logical categories, 2) analysis of data from each of the derived categories, and furthermore synthesized in final conclusions.

\section{Results and discussion}

In total 366 studies were identified using the above-mentioned inclusion criteria to provide an overview of the tools for measuring the professional identity of psychological help providers. The studies included following psychological help providers: art therapists including 4 arts therapies specialization (6 studies), nurses (136 studies), social workers (51 studies), psychotherapists (8 studies), psychiatrists (53 studies) and psychologists (112 studies). After manually screening the selected studies by two independent researcher according to the criteria - applied or described instrument to measure professional identity, only 21 were suitable for further assessment and the selected studies included nurses (14 studies), social workers (2 studies), psychiatrists (1 studies) and psychologists (4 studies).

For this study, the main category was the measurement tool of the professional identity which was either applied or described in the selected studies. From the studies included in this 
research the following information was obtained: name of the tool, author, year, source. As a result, a list of the professional identity tools was developed (Table 1).

\section{Table 1. Instruments for measuring the professional identity of psychological help providers}

\begin{tabular}{|c|c|c|c|c|}
\hline No. & $\begin{array}{c}\text { Name of the } \\
\text { tool }\end{array}$ & Author & Year & Source \\
\hline 1. & \begin{tabular}{|l|} 
Professional \\
Identity Index
\end{tabular} & $\begin{array}{l}\text { Manomenidis, } \\
\text { Kafkia, Minaisidou, } \\
\text { Tasoulis, Koutra, } \\
\text { Kospantsidou \& } \\
\text { Dimitriadou }\end{array}$ & 2017 & $\begin{array}{l}\text { Manomenidis, G., Kafkia, T., Minasidou, E., } \\
\text { Tasoulis, C., Koutra, S., Kospantsidou, A., \& } \\
\text { Dimitriadou, A. (2017). Is Self-Esteem Actually } \\
\text { the Protective Factor of Nursing Burnout? } \\
\text { International Journal of Caring Sciences, 10(3), } \\
\text { 1348-1359. }\end{array}$ \\
\hline 2. & $\begin{array}{l}\text { Professional } \\
\text { Identity Scale } \\
\text { in Counseling } \\
\text { (PISC) }\end{array}$ & Woo \& Henfield & 2015 & $\begin{array}{l}\text { Woo, H., \& Henfield, M.S. (2015). Professional } \\
\text { Identity Scale in Counseling (PISC): Instrument } \\
\text { Development and Validation, Journal of Counselor } \\
\text { Leadership and Advocacy, 2(2), 93-112. }\end{array}$ \\
\hline 3. & $\begin{array}{l}\text { Professional } \\
\text { Identity Status } \\
\text { Questionnaire } \\
\text { (PISQ-5d) }\end{array}$ & $\begin{array}{l}\text { Mancini, C } \\
\text { Panari \& T }\end{array}$ & 2015 & $\begin{array}{l}\text { Mancini, T., Caricati, L., Panari, C., \& Tonarelli, } \\
\text { A. (2015). Personal and social aspects of } \\
\text { professional identity: An extension of Marcia's } \\
\text { identity status model applied to a sample of } \\
\text { university students. Journal of Vocational } \\
\text { Behavior, 89, 140-150. }\end{array}$ \\
\hline 4. & \begin{tabular}{|l|} 
Professional \\
Identity Five \\
Factor Scale
\end{tabular} & $\begin{array}{l}\text { Tan, Molen \& } \\
\text { Schmidt }\end{array}$ & 2015 & $\begin{array}{l}\text { Tan, C. P., Van der Molen, H. T., \& Schmidt, H. } \\
\text { G. (2015). A measure of professional identity } \\
\text { development for professional education. Studies in } \\
\text { Higher Education, 42(8), 1504-1519. }\end{array}$ \\
\hline 5. & \begin{tabular}{|l|} 
Professional \\
Identity Scale \\
for Nursing \\
Students \\
(PISNS) \\
\end{tabular} & $\begin{array}{l}\text { o, Niu, Li, Yue } \\
\text { Liu }\end{array}$ & 2014 & $\begin{array}{l}\text { Hao, Y.F, Niu, H.J., Li, L.P., Yue, S.J., \& Liu, } \\
\text { X.H. (2014). Measurement of professional } \\
\text { identity in Chinese nursing students. } \\
\text { International Journal of Nursing Sciences, 1(2), } \\
\text { 137-144. }\end{array}$ \\
\hline 6. & \begin{tabular}{|l|} 
Nurses' \\
professional \\
values scale \\
REVISED \\
Korean version
\end{tabular} & $\begin{array}{l}\text { Moon, Kim, Kim, } \\
\text { Kim \& Lee } \\
\text { Original: (Weis \& } \\
\text { Schank, 2009) }\end{array}$ & 2014 & $\begin{array}{l}\text { Moon, S., Kim, D. H., Kim, E. J., Kim, Y.-J., \& } \\
\text { Lee, S. (2014). Evaluation of the validity and } \\
\text { reliability of the Korean version of the Nursing } \\
\text { Professional Values Scale--Revised. Nurse } \\
\text { Education Today, 34(3), 325-330. }\end{array}$ \\
\hline 7. & $\begin{array}{l}\text { Chinese } \\
\text { version of } \\
\text { nurses' self- } \\
\text { concept } \\
\text { questionnaire } \\
\text { (C-NSCQ) }\end{array}$ & 0 & 2012 & $\begin{array}{l}\text { Cao, X. Y., Liu, X. H., Tian, L., \& Guo, Y. Q. } \\
\text { (2012). The reliability and validity of the Chinese } \\
\text { version of nurses' self-concept questionnaire. } \\
\text { Journal of Nursing Management, 21(4), 657-667. }\end{array}$ \\
\hline 8. & $\begin{array}{l}\text { Nurses' } \\
\text { professional } \\
\text { values scale } \\
\text { REVISED } \\
\text { Turkish version }\end{array}$ & $\begin{array}{l}\text { Geçkil, Ege, Akın } \\
\text { \& Göz } \\
\text { Original: (Weis \& } \\
\text { Schank, 2009) }\end{array}$ & 2012 & $\begin{array}{l}\text { Geçkil, E., Ege, E., Akin, B., \& Göz, F. } \\
\text { (2012). Turkish version of the revised nursing } \\
\text { professional values scale: Validity and reliability } \\
\text { assessment. Japan Journal of Nursing Science, } \\
\text { 9(2), 195-200. }\end{array}$ \\
\hline 9. & $\begin{array}{l}\text { The Casey- } \\
\text { Fink Readiness } \\
\text { for Practice } \\
\text { Survey } \\
\end{array}$ & $\begin{array}{l}\text { Casey, Fink, } \\
\text { Jaynes, Campbell, } \\
\text { Cook \& Wilson }\end{array}$ & 2011 & $\begin{array}{l}\text { Casey, K., Fink, R., Jaynes, C., Campbell, L., } \\
\text { Cook, P., \& Wilson, V., (2011). Readiness for } \\
\text { practice: The senior practicum experience. Journal } \\
\text { of Nursing Education, 50(11), 646-652. }\end{array}$ \\
\hline 10. & $\begin{array}{l}\text { Professional } \\
\text { Identity and } \\
\text { Values Scale } \\
\text { (PIVS) }\end{array}$ & $\begin{array}{l}\text { Healey, Hays \& } \\
\text { Fish }\end{array}$ & 2010 & $\begin{array}{l}\text { Healey, A., Hays, D. G., \& Fish, J. (2010). A } \\
\text { grounded theory study of female counselor } \\
\text { educators on professional identity: Implica- tions }\end{array}$ \\
\hline
\end{tabular}




\begin{tabular}{|c|c|c|c|c|}
\hline & & & & $\begin{array}{l}\text { for wellness and training. Unpublished manuscript, } \\
\text { Sam Houston State University, Huntsville, TX. } \\
\text { Retrieved from: } \\
\text { Healey, A.C. \& Hays, D.G. (2012). A Discriminant } \\
\text { Analysis of Gender and Counselor Professional } \\
\text { Identity Development. Journal of Counseling and } \\
\text { Development: JCD; Alexandria, } 90(1) \text {. }\end{array}$ \\
\hline 11. & $\begin{array}{l}\text { Nurses' } \\
\text { professional } \\
\text { values scale } \\
\text { REVISED } \\
\text { Chinese version }\end{array}$ & $\begin{array}{l}\text { Lin \& Wang } \\
\text { Original: (Weis \& } \\
\text { Schank, 2009) }\end{array}$ & 2010 & $\begin{array}{l}\text { Lin, Y.H., \& Wang, L. S. (2010). A Chinese } \\
\text { version of the revised nurses professional values } \\
\text { scale: Reliability and validity assessment. Nurse } \\
\text { Education Today, 30(6), 492-498. }\end{array}$ \\
\hline 12. & $\begin{array}{l}\text { Professional } \\
\text { Self Identity } \\
\text { Questionnaire } \\
\text { (PSIQ) }\end{array}$ & $\begin{array}{l}\text { Crossley \& } \\
\text { Vivekananda- } \\
\text { Shhmidt }\end{array}$ & 2009 & $\begin{array}{l}\text { Crossley, J., \& Vivekananda-Schmidt, P. } \\
\text { (2009). The development and evaluation of a } \\
\text { Professional Self Identity Questionnaire to measure } \\
\text { evolving professional self-identity in health and } \\
\text { social care students. Medical Teacher, 31(12), } \\
\text { e603-e607. }\end{array}$ \\
\hline 13. & \begin{tabular}{|l|} 
The Nurse \\
Professional \\
Identity scale
\end{tabular} & Liu & 2009 & $\begin{array}{l}\text { Liu, L. (2009). Nurse professional identity and its } \\
\text { relationship with stress and burnout. Master's } \\
\text { thesis, The Second Military Medical University, } \\
\text { Shanghai, China. } \\
\text { Retrieved from: } \\
\text { Zhang, Y., Wu, J., Fang, Z., Zhang, Y., \& Wong, } \\
\text { F.K.Y. (2017). Newly graduated nurses' intention } \\
\text { to leave in their first year of practice in Shanghai: } \\
\text { A longitudinal study. Nursing Outlook, 65(2), 202- } \\
211 \text {. }\end{array}$ \\
\hline 14. & \begin{tabular}{|l|} 
Nurses \\
Professional \\
Values Scale \\
Revised NPVS- \\
R
\end{tabular} & Weis \& Schank & 2009 & $\begin{array}{l}\text { Weis, D., \& Schank, M.J. (2009). Development } \\
\text { and psychometric evaluation of the Nurses } \\
\text { Professional Values Scale-Revised. Journal of } \\
\text { Nursing Measuremen, 17(3), 221-31 }\end{array}$ \\
\hline 15. & $\begin{array}{l}\text { Professional } \\
\text { Identity and } \\
\text { Engagement } \\
\text { Scale (PIES), } \\
\text { The PIES } \\
\text { revised version }\end{array}$ & Puglia & 2008 & $\begin{array}{l}\text { Puglia, B. (2008). The professional identity of } \\
\text { counseling students in master's level CACREP } \\
\text { accredited programs (Doctoral dis- sertation, Old } \\
\text { Dominion University). Available from ProQuest } \\
\text { Dissertations and Theses database. } \\
\text { Retrieved from: } \\
\text { Healey, A.C., \& Hays, D.G. (2012). A } \\
\text { Discriminant Analysis of Gender and Counselor } \\
\text { Professional Identity Development. Journal of } \\
\text { Counseling and Development: JCD; Alexandria, } \\
90(1) \text {. }\end{array}$ \\
\hline 16. & $\begin{array}{l}\text { Macleod Clark } \\
\text { Professional } \\
\text { Identity Scale } \\
\text { (MCPIS-9) }\end{array}$ & $\begin{array}{l}\text { Adams, Hean, } \\
\text { Sturgis \& Clark }\end{array}$ & 2006 & $\begin{array}{l}\text { Adams, K., Hean, S., Sturgis, P., \& Clark, M.J., } \\
\text { (2006). Investigating the factors influencing } \\
\text { professional identity of first-year health and social } \\
\text { care students. Learning in Health and Social Care, } \\
5(2), 55-68 \text {. }\end{array}$ \\
\hline 17. & \begin{tabular}{|l|} 
The Social \\
Work Values \\
Survey
\end{tabular} & $\begin{array}{l}\text { Bradley, Maschi \& } \\
\text { Ward }\end{array}$ & 2006 & $\begin{array}{l}\text { Bradley, C., Maschi, T., \& Ward, K. (2006). Social } \\
\text { work values survey. New York, NY: Community } \\
\text { Research and Evaluation Collaborative. } \\
\text { Retrieved from: } \\
\text { Bradley, C., Maschi, T., O'Brien, H., Morgen, K., } \\
\text { \& Ward, K. (2012). Faithful but different: Clinical } \\
\text { social workers speak out about cerrer motivation } \\
\text { and professional values, Journal of Social Work } \\
\text { Education, 48(3), 459-477. }\end{array}$ \\
\hline
\end{tabular}




\begin{tabular}{|c|c|c|c|c|}
\hline 18. & $\begin{array}{l}\text { Clarity of } \\
\text { Professional } \\
\text { Identity }\end{array}$ & Dobrow \& Higgins & 2005 & $\begin{array}{l}\text { Dobrow, S. R., \& Higgins, M.C. } \\
\text { (2005). Developmental networks and professional } \\
\text { identity: a longitudinal study. Career Development } \\
\text { International, } 10(6 / 7), 567-583 \text {. }\end{array}$ \\
\hline 19. & $\begin{array}{l}\text { The Values } \\
\text { survey }\end{array}$ & $\begin{array}{l}\text { Rognstad, Nortvedt } \\
\text { \& Aasland }\end{array}$ & 2004 & $\begin{array}{l}\text { Rognstad, M.K., Nortvedt, P., \& Aasland, O. } \\
\text { (2004). Helping Motives in Late Modern Society: } \\
\text { values and attitudes among nursing students. } \\
\text { Nursing Ethics, 11(3), 227-239. }\end{array}$ \\
\hline 20. & $\begin{array}{l}\text { Nurses self- } \\
\text { concept } \\
\text { questionnaire } \\
\end{array}$ & Cowin & 2001 & $\begin{array}{l}\text { Cowin, L. (2001). Measuring nurses' self-concept. } \\
\text { Western Journal of Nursing Research, 23, 313-25. }\end{array}$ \\
\hline 21. & $\begin{array}{l}\text { The CRN's } \\
\text { Development of } \\
\text { Psychotherapis } \\
\text { ts Common } \\
\text { Core } \\
\text { Questionnaire } \\
\text { (DPCCQ) }\end{array}$ & $\begin{array}{l}\text { Society for } \\
\text { Psychotherapy } \\
\text { Research in New } \\
\text { Zealand, The } \\
\text { Collaborative } \\
\text { Research Network } \\
\text { Study (CRN) }\end{array}$ & 1989 & $\begin{array}{l}\text { Kazantzis, N., Calvert, S.J., Orlinsky, D.E., Rooke, } \\
\text { S., \& Ronan, K. (2010). Professional development } \\
\text { perceptions and activities of psychiatrists and } \\
\text { mental health nurses in New Zealand. The New } \\
\text { Zealand Medical Journal (Online), 123(1317), } \\
\text { 24-34. }\end{array}$ \\
\hline
\end{tabular}

According to the next steps of the narrative synthesis, the content of the professional identity's measurement instruments was analyzed to make a final synthesized conclusion for this study.

When analyzing the content of the professional identity measurement tools, 50 content items were identified to form the phenomenon of the professional identity. Furthermore, equal content items were merged into categories (Table 2).

Table 2. Items and category/criteria forming the content of professional identity

\begin{tabular}{|c|c|c|c|c|c|}
\hline $\begin{array}{l}\text { CATEGORY/ } \\
\text { CRITERION }\end{array}$ & $\begin{array}{l}\text { 1.Belonging to } \\
\text { a Profession }\end{array}$ & $\begin{array}{l}\text { 2.Professional } \\
\text { Self-esteem } \\
\end{array}$ & $\begin{array}{l}\text { 3.Professional } \\
\text { Self-reflection }\end{array}$ & $\begin{array}{l}\text { 4.Practice in } \\
\text { the Profession }\end{array}$ & $\begin{array}{l}\text { 5.Relationship } \\
\text { with colleagues }\end{array}$ \\
\hline ITEM & $\begin{array}{l}\text { 1.Belonging to } \\
\text { a profession } \\
\text { 2.Identification } \\
\text { with the } \\
\text { profession } \\
\text { 3.Professional } \\
\text { role }\end{array}$ & $\begin{array}{l}\text { 4.Professional } \\
\text { self-esteem } \\
\text { 5.Professional } \\
\text { self-image } \\
\text { 6.Professional } \\
\text { affirmation } \\
\text { 7.Professional } \\
\text { self-efficacy }\end{array}$ & $\begin{array}{l}\text { 8.Professional } \\
\text { self-reflection } \\
\text { 9.In depth } \\
\text { involvement in } \\
\text { the profession } \\
\text { 10.Thinking } \\
\text { about self in } \\
\text { terms of } \\
\text { profession } \\
\text { 11.Feedback } \\
\text { from } \\
\text { colleagues/peer } \\
\text { s } \\
\text { 12.Supervision } \\
\text { 13. Personal } \\
\text { psychotherapy } \\
\text { 14. Professional } \\
\text { identity } \\
\text { assessment }\end{array}$ & $\begin{array}{l}15 . \text { Practice in } \\
\text { profession } \\
16 . \\
\text { Development } \\
\text { of skills }\end{array}$ & $\begin{array}{l}\text { 17.Work, } \\
\text { relationship in } \\
\text { multidisciplinary } \\
\text { team } \\
\text { 18.Professional } \\
\text { communication } \\
\text { 19.Professional } \\
\text { relationship } \\
\text { 20.Professional } \\
\text { social skills }\end{array}$ \\
\hline $\begin{array}{l}\text { CATEGORY/ } \\
\text { CRITERION }\end{array}$ & $\begin{array}{l}\text { 6.Professional } \\
\text { Difference }\end{array}$ & $\begin{array}{l}\text { 7.Professional } \\
\text { Development } \\
\text { and } \\
\text { Improvement }\end{array}$ & 8.Networking & $\begin{array}{l}\text { 9.Distribution } \\
\text { of Knowledge }\end{array}$ & $\begin{array}{l}\text { 10.Engaging } \\
\text { Behavior }\end{array}$ \\
\hline
\end{tabular}




\begin{tabular}{|c|c|c|c|c|c|}
\hline ITEM & $\begin{array}{l}\text { 21.Professional } \\
\text { difference } \\
\text { 22.Professional } \\
\text { uniqueness } \\
\text { 23.Social } \\
\text { comparison }\end{array}$ & $\begin{array}{l}\text { 24.Continuing } \\
\text { education } \\
\text { (workshops, } \\
\text { seminars, } \\
\text { conferences) } \\
\text { 25.Professional } \\
\text { self-growth } \\
\text { 26.Reading } \\
\text { professional } \\
\text { books, journals } \\
\text { 27.Sertification } \\
\text { / accreditation }\end{array}$ & $\begin{array}{l}\text { 28.Working } \\
\text { with co- } \\
\text { therapist } \\
\text { 29.Shadowing } \\
\text { other } \\
\text { colleagues/peer } \\
\text { s } \\
\text { 30.Informal } \\
\text { meetings with } \\
\text { peers } \\
\text { 31.Professional } \\
\text { social support }\end{array}$ & $\begin{array}{l}\text { 32.Teaching } \\
\text { others } \\
\text { 33.Educating } \\
\text { society about } \\
\text { profession } \\
\text { 34.Supervisor, } \\
\text { mentoring for } \\
\text { colleagues } \\
\text { 35.Research in } \\
\text { the profession }\end{array}$ & $\begin{array}{l}\text { 36.Engaging } \\
\text { behavior } \\
\text { 37.Participation } \\
\text { in professional } \\
\text { events } \\
\text { (meetings, } \\
\text { conferences) } \\
\text { 38.Involvement } \\
\text { in a professional } \\
\text { association }\end{array}$ \\
\hline $\begin{array}{l}\text { CATEGORY/ } \\
\text { CRITERION }\end{array}$ & $\begin{array}{l}\text { 11.Integration } \\
\text { of Personal } \\
\text { Characteristics }\end{array}$ & $\begin{array}{l}\text { 12.Knowledge } \\
\text { About } \\
\text { Profession }\end{array}$ & $\begin{array}{l}\text { 13.Ethical and } \\
\text { Legal Aspects } \\
\text { of Profession }\end{array}$ & $\begin{array}{l}\text { 14.Knowledge } \\
\text {, Skills }\end{array}$ & $\begin{array}{l}\text { 15.Review of } \\
\text { Professional } \\
\text { Obligations } \\
\end{array}$ \\
\hline ITEM & $\begin{array}{l}\text { 39.Personal } \\
\text { spirituality } \\
\text { 40.Personal } \\
\text { values } \\
\text { 41.Personal } \\
\text { views } \\
\text { 42.Altruism, } \\
\text { desire to help }\end{array}$ & $\begin{array}{l}\text { 43.Knowledge } \\
\text { about the } \\
\text { development, } \\
\text { history, culture } \\
\text { of the } \\
\text { profession } \\
\text { 44.Following } \\
\text { the } \\
\text { development of } \\
\text { the profession } \\
\text { 45.Philosophy } \\
\text { of the } \\
\text { profession }\end{array}$ & $\begin{array}{l}\text { 46.Knowledge } \\
\text { of professional } \\
\text { legislation and } \\
\text { normative } \\
\text { documents } \\
\text { 47.Ethical } \\
\text { issues of } \\
\text { profession }\end{array}$ & $\begin{array}{l}\text { 48.Knowledge, } \\
\text { skills }\end{array}$ & $\begin{array}{l}\text { 49.Disappointme } \\
\text { nt in the } \\
\text { profession } \\
\text { 50.The desire to } \\
\text { leave the } \\
\text { profession, to } \\
\text { change the } \\
\text { profession }\end{array}$ \\
\hline
\end{tabular}

As a result, 15 categories were designed which included the items that form the content of professional identity. Moreover, these categories can be classified in line with theoretical approaches to the formation of the professional identity (Vignoles et al, 2011; Skorikov et al, 2011) - individual, relational and collective identity domains (Table 3).

Table 3. Categories in line with the theoretical approaches

\begin{tabular}{|l|l|l|}
\hline Individual identity domain & Relational identity domain & Collective identity domain \\
\hline Professional self-esteem & Relationship with colleagues & Distribution of knowledge \\
Professional self-reflection & Networking & Belonging to a profession \\
Continuing education & Engaging behavior & Practice in the profession \\
Integration of personal & & Knowledge about profession \\
characteristics & & Ethical and legal aspects of \\
Knowledge and skills & & profession \\
& & Professional difference \\
& & Review of professional \\
& & \\
\hline
\end{tabular}

According to this classification, most of the categories are regarding the collective and individual domains of the professional identity. Only three categories are classified with the relational domain. Nevertheless, this is also an important part of the professional development, 
especially when discussing the professional borders (Bortaščenoks et al, 2018; Ricou et al, 2018).

\section{Conclusions}

It can be concluded that professional identity is characterized in many aspects and there are various measuring tools to determine it. When studying professional identity, several parts of the content of professional identity are integrated in different measurement tools. Thus, there is no single and comprehensive tool for measuring the professional identity of psychological help providers.

As a result of this study 15 categories were identified as the content aspects of professional identity which could be applied in the further research studies regarding the professional identity measurement. Thus, several different aspects of the professional identity were identified therefore enriching the theoretical bases of this phenomenon. The categories and content items were identified after screening 366 research articles about professional identity therefore not only confirming the broad viewpoint of the professional identity, but also providing a structured system on the measurement of this phenomenon.

To increase understanding about professional identity it is necessary to continue to develop the content of this phenomenon. Measuring professional identity for groups of the psychological help providers could help to understand how the professional identity of each of the group could be described content wise and to increase the understanding about the professional boundaries.

\section{References}

Adams, K., Hean, S., Sturgis, P., \& Clark, J.M. (2006), Investigating the factors influencing professional identity of first-year health and social care students. Learning in Health and Social Care, 5, 55-68.

Bortaščenoks, R., Purvlīce, B., Mārtinsone, K., Rancāns, E., Mihailovs, J.I., \& Ķīvīte - Urtāne, A. (2019). Psychological Help in Latvia - Regulatory Framework. Society. Integration. Education. Proceedings of the International Scientific Conference. 3, May 24th-25th, 2019. 21-36. DOI: http://dx.doi.org/10.17770/sie2019vol7.3806

Bradley, C., Maschi, T., O'Brien, H., Morgen, K., \& Ward, K. (2012). Faithful but different: Clinical social workers speak out about cerrer motivation and professional values, Journal of Social Work Education, 48(3), 459-477.

Casey, K., Fink, R., Jaynes, C., Campbell, L., Cook, P., \& Wilson, V., (2011). Readiness for practice: The senior practicum experience. Journal of Nursing Education, 50(11), 646-652. DOI: 10.3928/01484834-20110817-03

Cao, X. Y., Liu, X. H., Tian, L., \& Guo, Y. Q. (2012). The reliability and validity of the Chinese version of nurses' self-concept questionnaire. Journal of Nursing Management, 21(4), $657-667$.

Cowin, L., Johnson, M., Wilson, I., \& Borgese, K. (2013). The psychometric properties of five Professional Identity measures in a sample of nursing students. Nurse Education Today, 33(6), 608-613.

Cowin, L. (2001). Measuring nurses' self-concept. Western Journal of Nursing Research, 23, $313-25$. 
Crossley, J., \& Vivekananda-Schmidt, P. (2009). The development and evaluation of a Professional Self Identity Questionnaire to measure evolving professional self-identity in health and social care students. Medical Teacher, 31(12), e603-e607.

Dobrow, S., \& Higgins, M. (2005). Developmental networks and professional identity: A longitudinal study. Career Development International, 10(6/7), 567-583,587.

Geçkil, E., Ege, E., Akin, B., \& Göz, F. (2012). Turkish version of the revised nursing professional values scale: Validity and reliability assessment. Japan Journal of Nursing Science, 9(2), 195-200.

Hao, Y.F., Niu, H.J., Li, L.P., Yue, S.J., \& Liu, X.H. (2014). Measurement of professional identity in Chinese nursing students. International Journal of Nursing Sciences, 1(2), 137144.

Healey, A.C. \& Hays, D.G. (2012). A Discriminant Analysis of Gender and Counselor Professional Identity Development. Journal of Counseling and Development: JCD; Alexandria, 90(1). DOI: https://doi.org/10.1111/j.1556-6676.2012.00008.x

Healey, A.C., Hays, D. G., \& Fish, J. (2010). A grounded theory study of female counselor educators on professional identity: Implica- tions for wellness and training. Unpublished manuscript, Sam Houston State University, Huntsville, TX.

Kazantzis, N. Calvert, S.J, Orlinsky, D.E., Rooke, S., \& Ronan, K. (2010). Professional development perceptions and activities of psychiatrists and mental health nurses in New Zealand. The New Zealand Medical Journal (Online), 123(1317), 24-34.

Kegan, R. (1984). The Evolving Self: Problem and Process in Human Development. The Canadian Journal of Sociology, 9(3), 357-359.

Lin, Y.H., \& Wang, L. S. (2010). A Chinese version of the revised nurses professional values scale: Reliability and validity assessment. Nurse Education Today, 30(6), 492-498.

Mancini T. Caricati L. Panari C. \& Tonarelli A. (2015). Personal and social aspects of professional identity: An extension of Marcia's identity status model applied to a sample of university students. Journal of Vocational Behavior, 89, 140-150.

Manomenidis, G., Kafkia, T., Minasidou, E., Tasoulis, C., Koutra, S., Kospantsidou, A., \& Dimitriadou, A. (2017). Is Self-Esteem Actually the Protective Factor of Nursing Burnout? International Journal of Caring Sciences, 10(3), 1348-1359.

Mārtinsone K. \& Sudraba V. (2019). Veselības psihologija. Teorijas un prakses starpdisciplinārā perspektīva. Rīga: Rīgas Stradina universitate.

Moon, S., Kim, D. H., Kim, E. J., Kim, Y.-J., \& Lee, S. (2014). Evaluation of the validity and reliability of the Korean version of the Nursing Professional Values Scale-Revised. Nurse Education Today, 34(3), 325-330.

Purvlīce, B., Mārtinsone K., Pipere A., \& Bortaščenoks R. (2018). Identity of Professional Groups Providing Psychological Help: a Literature Review. Society. Integration. Education. Proceedings of the International Scientific Conference. 3, May 25th-26th, 2018, 229-239. DOI: http://dx.doi.org/10.17770/sie2018vol1.3296

Ricou, M., Cordeiro, S.A., Franco, A.R., \& Costa-Lobo, C. (2018). The Identity of Psychology. The Psychologist: Practice \& Research Journal, 1-15.

Rognstad, M.-K., Nortvedt, P., \& Aasland, O. (2004). Helping Motives in Late Modern Society: values and attitudes among nursing students. Nursing Ethics, 11(3), 227-239.

Skorikov, V.B., \& Vondarcek, F.W. (2011). Occupational identity. In: Schwartz SJ, Luyckx K, Vignoles VL, eds. Handbook of Identity Theory and Research. New York, NY: Springer.

Spurgeon, S. (2012). Counselor identity - a national imperative. Journal of Professional Counseling, Practice, Theory, \& Research, 39(1), 3.

Tan, C. P., Van der Molen, H. T., \& Schmidt, H. G. (2015). A measure of professional identity development for professional education. Studies in Higher Education, 42(8), 1504-1519. 
Vignoles, V.L., Schwartz, S.J., \& Luyckx, K. (2011). Toward an integrative view of identity. In: Schwartz SJ, Luyckx K, Vignoles VL, eds. Handbook of Identity Theory and Research. New York, NY: Springer.

Weis, D., \& Schank, M. (2009). Development and psychometric evaluation of the Nurses Professional Values Scale--Revised. Journal of Nursing Measurement, 17(3), 221-231.

Woo, H., \& Henfield, M.S. (2015). Professional Identity Scale in Counseling (PISC): Instrument Development and Validation, Journal of Counselor Leadership and Advocacy, 2(2), 93-112.

World Health Organization: Fact Sheets "Mental health: strengthening our response", 2018, retrieved from https://www.who.int/news-room/fact-sheets/detail/mental-healthstrengthening-our-response.

Zhang, Y., Wu, J., Fang, Z., Zhang, Y., \& Wong, F.K.Y. (2017). Newly graduated nurses' intention to leave in their first year of practice in Shanghai: A longitudinal study. Nursing Outlook, 65(2), 202-211. 